\title{
Artificial Intelligence for the Advancement of Lunar and Planetary Science and Exploration
}

July 15

A White Paper submitted to the

Planetary Science and Astrobiology Decadal Survey 2023-2032

\author{
Lead Author \\ Indhu Varatharajan \\ DLR Institute of Planetary Research, Rutherfordstr. 2, Berlin 12489, Germany \\ Email: indhu.varatharajan@dlr.de
}

Contributing Authors: Daniel Angerhausen ${ }^{1}$, Eleni Antoniadou ${ }^{2}$, Valentin Bickel ${ }^{1,2,3}$, Mario D’Amore ${ }^{4}$, Michele Faragalli ${ }^{5}$, Ignacio López-Francos ${ }^{2,6}$, Abhisek Maiti ${ }^{7}$, Ross Potter ${ }^{8}$, Carl Shneider ${ }^{9}$, Shashwat Shukla ${ }^{7}$, Frank Soboczenski ${ }^{2,10}$, Nishan Srishankar ${ }^{11}$, Ryan Watkins ${ }^{12}$

Co-Signers: Caitlin Ahrens ${ }^{13}$, Brendan A. Anzures ${ }^{8}$, Abigail Azari ${ }^{14}$, Jennifer G. Blank ${ }^{6,15}$, Aditya Chopra ${ }^{16,17}$, Jörn Helbert ${ }^{4}$, Jose M. Hurtado, Jr. ${ }^{18}$, Devanshu Jha ${ }^{19}$, Nandita Kumari ${ }^{20}$, Alexander Lavin ${ }^{21}$, Ying Liao ${ }^{22}$, Alison B Lowndes ${ }^{23}$, Ben Moseley ${ }^{2,24}$, James Parr ${ }^{2}$, Kaizad Raimalwala $^{5}$, Vidhya Ganesh Rangarajan ${ }^{25}$, Roger Stabbins ${ }^{26}$
Affiliations:
${ }^{1}$ ETH Zurich
${ }^{2}$ Frontier Development Laboratory
3 Max Planck Institute for Solar System
Research
${ }^{4}$ DLR Institute of Planetary Research
${ }^{5}$ Mission Control Space Services Inc
${ }^{6}$ NASA Ames Research Center
${ }^{7}$ Faculty ITC, University of Twente, The
Netherlands
${ }^{8}$ Brown University
9 National Center for Mathematics and
${ }^{13}$ University of Arkansas
${ }^{14}$ UC Berkeley
${ }^{15}$ Blue Marble Space Institute of Science
${ }^{16}$ University of Groningen
17 The Australian National University
${ }^{18}$ The University of Texas at El Paso
${ }^{19}$ MVJ College of Engineering, India
${ }^{20}$ Stony Brook University
${ }^{21}$ Augustus Intelligence \& Latent Sciences
${ }^{22}$ National Central University, Taiwan
${ }^{23}$ NVIDIA
Computer Science (CWI), The Netherlands
${ }^{10}$ SPHES, King's College London, UK
${ }^{11}$ Worcester Polytechnic Institute
${ }^{12}$ Planetary Science Institute
${ }^{24}$ Department of Computer Science, University of Oxford
${ }^{25}$ University of Western Ontario
${ }^{26}$ Natural History Museum (London) 


\section{Executive Summary}

Planetary data obtained from telescope, orbital, and landed missions are enabling the lunar and planetary community to answer important science questions regarding the origin, evolution, surface mineralogy, volatile resources, volcanic processes, and surface processes of a planet. Artificial intelligence (AI)-driven methods have the potential to reduce the manual labor time needed for data processing and analysis and aid ongoing missions with rapid real-time data analysis. This white paper focuses on: areas of lunar and planetary research that could benefit from AI-driven approaches, the need for open source training/validating/testing AI and machine learning (ML) datasets, and the importance of cross-collaboration between academia and industries for supporting next-gen lunar and planetary science and exploration.

\section{Introduction}

Since human exploration of the Moon in the 1960s, the lunar community has benefited from a series of successful missions including flybys, orbiters, landers (crewed and robotic), rovers, and impactors (Fig. 1). These missions, along with telescope observations, have contributed a significant amount of data to address questions including how the Moon formed and evolved through time, what its surface processes and resources are, and the nature of the chemical composition of its surface and deep interior. Various experimental data on the physical properties, chemistry, and mineralogy of returned samples and meteorites provide us with ground-truth data at a finer resolution which further serves as ground-truth for remote sensing observations.

Over the past decades of NASAs inner solar system exploration, data obtained from the Moon alone accounts for $\sim 76 \%$ (Fig. 2a). Many lunar orbital spacecraft of the past and present carried imaging cameras and spectrometers (including multispectral and hyperspectral payloads), as well as a large variety of other passive and active instruments. For example, NASAs Lunar Reconnaissance Orbiter (LRO) has been operating for 10+ years, providing 1206 TB of lunar data, which amounts to $\sim 99.5 \%$ of the total data contributed by NASA built instruments (Fig. 2b). Given recent advances in instrument and communication capabilities, the amount of data returned from spacecraft is expected to continue rising quickly.

Irrespective of this huge amount of available data, many important questions regarding lunar and planetary science remain unanswered. For example, the lunar community still faces challenges to address major science questions $[3,4,5]$ such as:

- Is the Moon still geologically active?

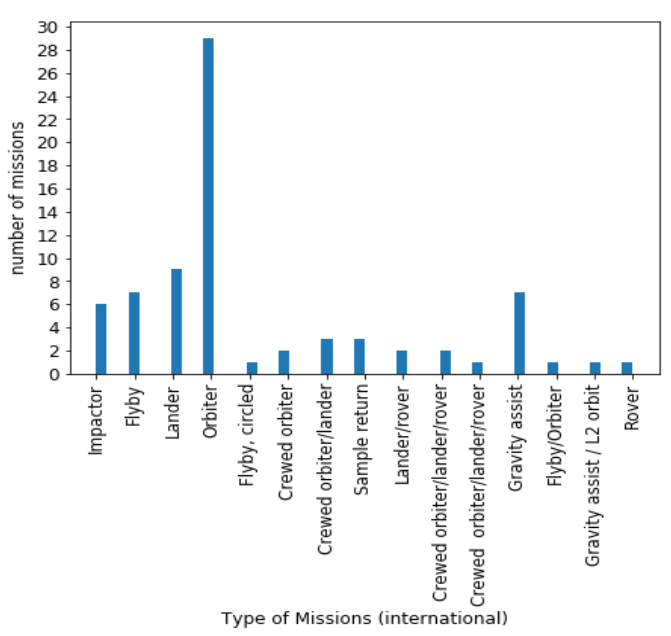

Figure 1. Total number of successful missions (including currently operating ones) to the Moon. The data webscrapped from [1].
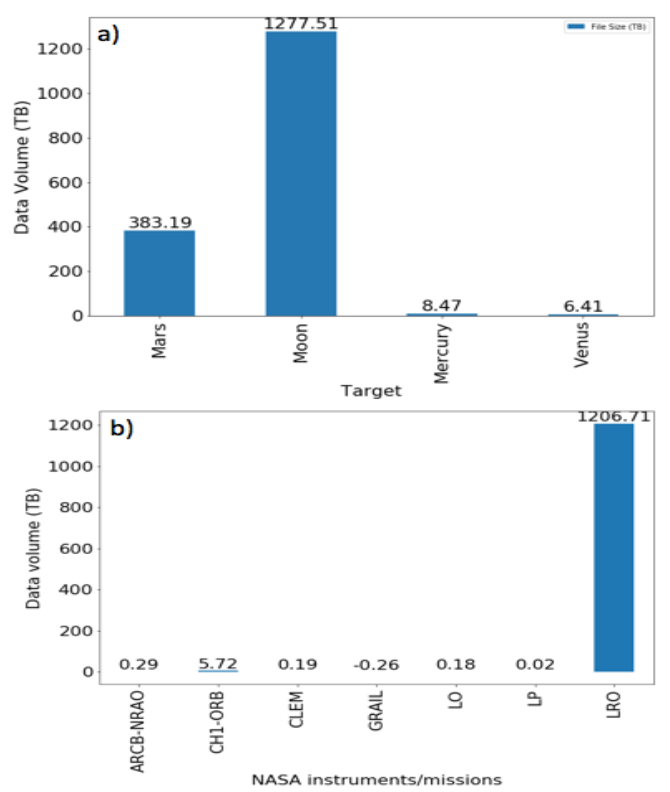

Figure 2. a) Planetary Data Volume acquired by NASA missions for inner solar system targets. b) Total data obtained by Moon Mineralogy Mapper (M3) instrument onboard ISRO's Chandrayaan-1 (CH1-ORB), Clementine (CLEM), Gravity Recovery and Interior Laboratory (GRAIL), Lunar Orbiter (LO), Lunar Prospector (LP), and LRO. Data webscrapped from [2]. 
- What is the total amount of exogenic volatiles at the lunar poles, their stability, as well as their vertical and lateral distribution?

- What is the origin of lunar swirls?

- What is the distribution and extent of lunar caves and lava tubes?

- What is the influence of physical processes on volatiles at mining scales?

- What is the nature and distribution of terrestrial meteorites present on the Moon?

- What is the distribution of volatiles in the (deep) lunar interior?

The next-generation of lunar exploration will include a cis-lunar station (Deep Space Gateway), small-sat/cube-sat missions, crewed missions (Artemis), In-Situ Resource Utilization (ISRU)-based missions (VIPER) etc. that will further generate significant amounts of data that will help to address the above questions, as well as many others. As data volume increases, the processing and analysis of these data becomes more challenging using traditional approaches. For this decade and those to come, more efficient and faster data analysis methods need to be developed and implemented.

On the one hand, complete utilization of the currently available legacy datasets is vital in order to effectively plan and execute future missions. This can be facilitated by applying AI/ML-based methods that are capable of providing detailed, large-scale mapping of the lunar surface and rapid data analysis, as well as other advantages. Additionally, AI and ML-methods will be useful for operating and guiding robots and humans "on the fly", during upcoming missions, e.g. by improving rover path planning, with real-time big data analysis, or with anomaly detection procedures.

This white paper highlights potential components of AI and ML that could help accelerate the future exploration of the Moon and other planetary bodies. The following discussion is divided into three main sections:

1) AI/ML-based approaches for lunar and planetary surface science and exploration

2) The need for open source availability of training, validation, and testing datasets for AI-ML based approaches

3) Bridging the gap between industry and academia for advancing AI-ML based research

\section{AI/ML-based approaches for lunar and planetary surface science and exploration}

Lunar orbiters provide huge volumes of data allowing questions regarding surface processes, space weathering, global mineralogy, chemical composition of the lunar interior, volcanic activity, impact cratering, space weathering and more to be addressed.

Here we showcase a few examples of scientific applications that could potentially benefit from AI-ML based approaches. This list is not complete but is intended to highlight the potential benefit of ML and AI for lunar and planetary science and exploration.

\subsection{Analysis of lunar geomorphology and surface activity from orbit}

The lunar surface consists of a variety of geomorphological features like impact craters, lunar swirls, and volcanic structures including vents, rilles, domes, lunar caves and lava tubes. Detailed mapping of these features is important but is very tedious and time-consuming. For example, crater counting allows for the estimation of surface ages, but requires manual labeling of craters over a wide range of diameters. As another example, the mapping of mass wasting features like rockfalls potentially allows for the localization of recent shallow moonquake epicenters, but rockfalls are very difficult to locate in satellite imagery. Tasks like these greatly benefit from ML and AI-enabled automation, for example by using convolutional neural networks, i.e. tools from AI fields like computer vision [eg., 6,7].

Potential, additional AI-ML based approaches for remote mapping of the lunar surface could include:

Super-resolution: Multiple instruments onboard various lunar orbiters and rovers provide datasets of varying spatial resolutions. In most cases, very high resolution (targeted) datasets 
are not globally available compared to relatively low-resolution imaging cameras that have global coverage. Super resolution (SR) is a technique where a low-resolution image is taken as an input and is upscaled to a higher resolution as the output. AI-driven SR approaches have the potential to provide globally uniform and targeted high-resolution datasets which could aid in selecting landing sites for next-gen lunar exploration [e.g., 8].

Surface feature detectors: Specifically, trained detectors (such as convolutional neural networks) could map features of interest on a global scale, e.g., impact craters $<1 \mathrm{~km}$ diameter [e.g., 9], rockfalls and other mass wasting features [e.g., 10], or fresh impact craters, among numerous others.

\subsection{Lunar orbital spectroscopy and surface ground truth for global resource mapping}

Spectroscopy gives the opportunity to study the surface mineralogy of any planetary body from orbit. Spectrometers with wide spectral range, very-high spectral $(<20 \mathrm{~nm})$ and spatial resolution, and with repeated orbital coverage help us to map the surface mineralogy and resources of planets in greater detail. Various spectral ranges tell different stories and properties of the surface. For example, VIS-IR spectroscopy informs the distribution of $\mathrm{Fe}, \mathrm{Ti}, \mathrm{Mg}$, and $\mathrm{Ca}$ rich minerals for a rocky planet's igneous and sedimentary phases including volatiles, whereas thermal IR spectroscopy reveals the Si-O abundance on the bulk mineralogy. Spectroscopy therefore aids in developing planetary resource maps, including volatiles. These and other chemical signatures also serve to trace and to constrain critical and changing space weather activity [e.g., 11]. By carefully building training datasets documenting the spectral behavior of various lunar analogues under simulated laboratory conditions representing lunar surface environment, AI-ML based maps can effectively map the global material characterization [e.g., 12, 13], mineral distribution, and their abundances from orbit. Thereby, the integrated approach of laboratory + field analogue + remote + in situ spectroscopy leads to efficient exploration of minerals and resources on the exposed lunar surface.

Among data generation by orbital payloads, multi- and hyper- spectral imaging cameras are among the largest producers of data in any mission $[14,15]$. Global surface science from these hyperspectral imagers by integrating the laboratory spectroscopy of minerals in the particular planetary environment are not yet widely exploited by the lunar community. AI-ML powered hyperspectral imaging solutions on surface-based rovers and landers will further improve traversing and resource exploitation over the lunar surface.

Potential, additional AI-ML based approaches for lunar spectroscopy and surface science include:

Integrated spectroscopy: Combining multi-mission multi- and hyper- spectral datasets covering the entire spectral range $(0.3-14 \mu \mathrm{m})$ provides a unique way of exploring lunar resources for its nature and abundance.

Resource mapping: Determine the mineral and volatile composition of the soil by training a ML model on reflectance spectra [e.g., 13]. Then deploying the ML model onboard a surfacebased mobile robot to enable automatic characterization of environment and identification of resources of interest, such as volatiles [e.g 16], radiogenic elements, and metals.

\subsection{Orbital Radar Science for the analysis of volatile resources}

Topographic depressions at the lunar poles (or the poles of other planetary bodies such as Mercury) receive no direct sunlight at all, presenting a unique geologic setting for science and exploration. In sharp contrast, specific topographic rises along, for example, crater rims are illuminated by near-continuous sunlight. Permanently shadowed regions (PSRs), possessing such geomorphological characteristics, have the potential to harbor near surface, high-grade volatile reservoirs on the Moon. One of the orbital instruments that possess a unique capability to 'probe in the dark', owing to its active source, is imaging radar. Radar mapping therefore helps to characterize distinct geologic features contained at surface and subsurface levels, and are capable of identifying the physical state, distribution, and overall abundance of lunar 
volatiles such as water-ice and helium-3 [17]. Understanding the interaction of radar waves with lunar and planetary surfaces is a very complex phenomenon. From physical electromagnetic models, the sensitivity of radar backscatter with surface parameters is highly nonlinear and thus, (semi-) empirical methods fail to operate and yield reliable results [17]. This is mainly caused by the limitations in procuring the ground truth. Recently, machine learning has provided robust methodologies and automatic techniques in radar science given its capability to handle multivariate, highly complex and non-linear data [17,18]. AI-based approaches for the evaluation of radar responses relevant to surface and sub-surface volatile sensing therefore play an important role in prospecting target regions of ISRU.

Potential, additional AI-ML based approaches for radar-driven mapping of lunar polar volatiles could include:

Retrieval of Physical Properties: Electromagnetic forward models coupled with ML-based inversion algorithms can be used for the retrieval of physical properties that influence the capability of regolith to harbor near-surface reservoirs of volatiles [e.g., 17].

Global Mapping: Robust ML-based approach in creating global and polar dielectric constant maps of the lunar surface allow identifying and characterizing the concentration of volatiles at mining scales using complex data of radar backscatter [18].

\subsection{Surface-based exploration}

Rovers and landers are an integral part of next-gen lunar and planetary exploration strategies [19]. For example, one of the major challenges of lunar surface exploration is the length of one lunar day, which roughly lasts a full 14 Earth days. For solar-powered rovers and landers, this 14-day cycle poses a unique challenge for surviving multiple lunar day-night cycles. Therefore, the entire process including autonomous landing, rover traversing/navigation, data measurements and analysis, and surface telecommunication must all take place in a very short timescale for multiple measurements covering large surface areas. Additionally, more efficient payload instrument data analysis is critical to the science return of the mission.

Potential AI-ML based approaches toward surface-based lunar exploration include:

Landing site selection: This is the most crucial step for surface-based exploration where possible applications can range from solar system activity and planetary weather analysis to surface or subsurface analysis. For example, during landing site analysis of ISRU missions at the lunar south pole, the most challenging task is to find a relatively smooth patch of the lunar surface without larger boulders and craters in the most rugged lunar terrain. AI/ML-based solutions can combine multiple datasets, possibly fusion datasets of high-resolution camera images and radar datasets, can aid in faster and smarter selection of landing sites for successful missions. In addition, ML-based pipelines for low-light image processing (i.e. denoising) could aid on this task by revealing the morphology and topology of those areas perpetually in shadow.

Landing+Traversing+Telecommunication: These three roles are the most integral part to rate the success of the mission. Failure of any one process, can lead to failure of the entire mission. For example, the failed landing of Vikram at the lunar south pole led to failure of testing the first AI-powered lunar rover (Pragyan) by ISROs Chandrayaan-2 [20]. Without global positioning systems orbiting the Moon, navigation and telecommunication between multiple rovers, landers, and orbiters poses the next level of challenge. This drives the need for AI-based absolute localization solutions, in which ground view images from the cameras onboard a rover can be warped to obtain a bird's eye view (BEV) of the ground and be matched with satellite imagery [e.g., 21].

Rover Driving Technologies: To date, rover driving technologies mostly depend on surface imagery to plan further actions, but this is limited by the rover's vision. Moreover, due to the variations in terrain and illumination followed by camera viewing constraints, rovers on the ground are naturally limited in their ability, leading to uncertain and incorrect decisions if not administered. With the advancement of AI in autonomous driving applications, its capability 
can be applied to a novel setting, i.e., for planetary rover exploration. For example, deep reinforcement learning (RL) techniques has been optimized for hopping rovers in challenging space environments using least squares policy iteration [22, 23]. For a robot to successfully operate in a given environment it must make sense of it, plan its actions and execute those plans using some means of actuation, while using feedback on success. RL introduces reward functions which allows a robot to maximize given correct actions taken. In order to allow the algorithm to "learn to plan", a value iteration algorithm used inside RL can be implemented by binding it to the map of planetary terrain along with the available state transitions based on the obstacles present [24]. For assisting the rover to navigate using deep learning algorithms, the need for specific real-time data can produce reliable and robust results in the context of navigation planning. Given the vast amounts of data from terrain images as well as any synthetic data and human annotation, learning-based approaches could be used to identify and segment terrain types [25,26], generate steering commands through deep visual odometry or even optimize paths to minimize time/ risk factors while maximizing the science objective.

Pinpoint Landing: NASA Commercial Lunar Payload Service (CLPS) landers will soon be routinely delivering science and technology payloads to the lunar surface. This will require a high degree of landing accuracy, especially at the poles due to the challenging conditions there. Whereas previous missions were satisfied with a landing accuracy of kilometres [27], the polar region will require an accuracy within a few hundred meters and very low descent rates for improved safety and probability of mission success. Most current powered descent phase guidance and control systems use two separate and independently optimized systems for guidance and control where the guidance system generates a trajectory that specifies the lander's target state as a function of time and the trajectory is then passed to a control system that tracks the trajectory by determining which thrusters to fire and at what thrust level. An approach to improve the landing accuracy is using Reinforcement Learning algorithms to learn a global policy to map the guidance, navigation, and control (GNC) system's estimate of the lander's state directly from the commands specifying thirst levels for each engine [28]. Deep RL techniques embedded in GNC systems have shown promising results that may lead to improved performance, accuracy, speed, minimum thruster propellant, and increased reliability for autonomous landings. An advantage of RL over traditional engineering GNC systems is its ability to adapt to unknown situations and circumstances that are difficult to foresee or cumbersome to account for manually. Consequently, challenges in autonomous navigation and control are natural fits for RL as soon as uncertainty arises and robustness is of importance.

\section{The need for open source availability of training, validation, and testing datasets for AI-ML- based approaches}

The digital universe in 2020 is projected to reach 40 zettabytes (ZB) - 5,200 GB of data for every person on Earth and amounts to 40 times more bytes than there are stars in the observable universe [29]. Open-source datasets that are readily available constitute a critical component for the development of ML and AI research, while enabling the cost-effective access of open data for scaled algorithmic training by citizen scientists. Moreover, open data in lunar research support multidisciplinarity and accelerated innovation in ML by providing increased access to scientists from diverse fields with different methodological approaches. In the context of lunar research, the term data includes photographic data, observation data, metadata, algorithms, models, as well as surface mineralogic, geophysical and geochemical data. It is therefore important to ensure that high standards of data integrity are upheld for ML tools and a harmonized framework for technical guidelines is created as new sensing instruments are deployed and technology progresses.

The operationalization of lunar data access requires data trusts, contractual agreements, and special cooperation between the different space agencies, academia and industrial partners so that data governance can be formulated. In order to maintain high quality data, the 
framework's performance must be audited, and guidelines for universal use must be developed. Moreover, cybersecurity risks that may appear during implementation need to be assessed to ensure the safety and sustainability of the open access framework. Finally, technology policies govern the requirements for dataset storage diversity. For example, the U.S. Congress and the White House implemented the Federal Data Strategy [30], a federal mandate that specifies the channels where data can be released and the ways they can be harvested by other sites. In addition, a significant amount of scientific data from lunar missions is created or analyzed by academic labs, which are funded by institutional grants. Under this premise, data analyzed by university labs are stored in scientific domain archives. Only a tiny fraction of the world's data is open access, and the same applies to data from space exploration missions despite recent efforts from several space agencies to render their digital sharing platforms open access (such as NASA [31,32], and ESA [33]).

While there are areas in ML that focus on how well a model can be trained with as little data as possible (e.g. one-shot learning) there is the general need for AI-based solutions for large training data sets specific to the particular problem. In most cases, curating training sets (assembly, preprocessing, merging etc) take the longest manual labor time. With continuing applications of AI-related solutions for lunar science and exploratory missions, it would be ideal to create a platform that makes the training datasets available open-source and further allow the lunar and planetary research community to share and evaluate the existing pre-trained models. The Moon is the target of international interest which increases the AI-based research aspects of various types of datasets. Github [34] and Gitlab [35] are still the popular ways to share algorithms, however they do not allow large data volume files. Appropriate funding for dedicated open source web portals, such as Planetary Data System (PDS) [e.g., 2], would allow researchers to submit their AI-based lunar research work along with their algorithm and training datasets along with an API that enables faster download of huge datasets.

\section{Bridging the gap between industry and academia for advancing AI-ML based research}

The upcoming decade of lunar exploration will involve commercial participation advancing and expediting next-gen lunar exploration. The NASA CLPS programme is driving industry participation by contributing small rovers and landers to explore lunar polar resources, test ISRU-concepts, and address science objectives in support of the Artemis program [36]. In addition to technological advances, these programmes highlight the need to further bridge the gap between academia and industry for mission planning and landing site selection, choosing suitable instrument sets for identifying and mapping lunar resources, and data analysis. These mission phases will benefit from the support of AI-ML-driven research.

The collaborative nature of the academia-industry relation in the field of AI has a longstanding and impactful tradition, from the 1956 Dartmouth Conference [37], where Dartmounth, Harvard, IBM and Bell Labs joined forces, to the Autonomous Driving Grand DARPA Challenge [38], the Semantic Web [39], the MIT-IBM Watson AI Lab [40] and countless in-market experimentations. This synergy produces publications, patents and software, that often results in licensing revenue streams as well as facilitates the crosspollination of AI industry laboratories such as Google, Microsoft, NVIDIA, Facebook, Amazon and IBM, through sabbaticals, faculty visiting fellowships and academic consortiums. The most important advantages of this unique academic-industry partnership are the agile access to deep expertise, noncompetitive peer research, increased diversity and multidisciplinarity, scaling productivity and accelerated timeline for success metrics.

Research programmes such as Frontier Development Laboratory (FDL), a NASA partner, is an AI research accelerator that brings multiple teams of researchers together with the aim of AI-based solutions to multiple challenges that benefit space science, medicine, and all humankind. The unique nature of this research accelerator is the fusion of the strengths of individuals from academia, government, and industry such as NASA, SETI, NVIDIA, Google 
Cloud, USGS, Lockheed Martin and more (see [41] for FDL 2020 program and collaborators), who involved from machine learning and other domains to develop unique solutions to advanced problems such as lunar resource mapping [42]. Industry partners such as Google Brain and NVIDIA fund researchers and facilitate the use of industry's high-performance cloud computing resources to support AI-based approaches in solving problems related to planetary [e.g., 43] and exoplanetary studies [e.g., 44]. Creating additional interdisciplinary, interindustry platforms to overcome the computational and technical challenges will greatly enhance AI-based lunar and planetary research over the next decade.

\section{Conclusions}

AI- and ML- -driven tools and methodologies have the potential to support the lunar and planetary science community, effectively exploit the currently available datasets, and help prepare for the next decade of science and exploration. AI-based solutions are capable of improving existing, or producing new unique datasets using techniques such as super resolution, anomaly detection, and data fusion, shedding new light on the processes that shaped - and still shape - the solar system. Incorporating and advancing AI- and ML-based methods and approaches in community and agency workflows will enable NASA and other agencies to advance respective science domains, pursue and achieve critical mission goals and visions. Broader discussion supporting AI and ML in planetary sciences are additionally discussed in other white papers to this decadal survey focusing on integration of ML to planetary science, data structures, and automation.

References: [1] Lunar Exploration Timeline and Missions, LPI. [2] Moon Holdings, PDS Geosciences Node [3] National Research Council. (2007) The scientific context for exploration of the Moon. [4] LEAG Specific Action Team Report (2017) Advancing Science of the Moon. [5] The Lunar Exploration Roadmap (2016), Exploring the Moon in the 21st Century, LEAG. [6] Salih, A. L. et al (2017) Automatic detection of secondary craters and mapping of planetary surface age... [7] Bickel, V. T. et al (2019) Automated Detection of Lunar Rockfalls Using a Convolutional Neural Network. [8] Bulyshev, A et al (2011) A super-resolution algorithm for enhancement of FLASH LIDAR data. [9] Silburt, A., et al., (2019) Lunar crater identification via deep learning [10] Bickel, V.T. et al. (2020) Impacts drive lunar rockfalls over billions of years. [11] Prabal Saxena et al (2019) Was the Sun a Slow Rotator? Sodium and Potassium Constraints from the Lunar Regolith. [12] Kodikara, G.R.L. and L.J. McHenry (2020) Machine learning approaches for classifying lunar soils. [13] Cambioni, S., et al. (2019) Constraining the thermal properties of planetary surfaces using machine learning... [14] C. M. Lee et al. (2015) An introduction to the NASA Hyperspectral InfraRed Imager (HyspIRI) mission and preparatory activities. [15] M. B. Stuart et al. (2019) Hyperspectral imaging in environmental monitoring...[16] K.M. Cannon and D.T. Britt, (2020) A geologic model for lunar ice deposits at mining scales. [17] Shukla S., et al., (2020) Modelling the Physical Nature of Lunar Regolith at S-Band and L-Band Wavelengths using the Chandrayaan-2 DFSAR and LRO Mini-RF Radars [18] Shukla S., et al (2020) Mini-RF Global and Polar S-Band Maps of the Variation in the Moon's Regolith Dielectric Constant. [19] VIPER, Artemis, Mars 2020 Perseverence Rover. [20] Chandrayaan 2 - orbiter, rover, lander. [21]. Wu, B., et al. (2019) Absolute Localization Through Orbital Maps and Surface Perspective Imagery: A Synthetic Lunar Dataset and Neural Network Approach. [22] Pflueger M., et al (2019) Rover-IRL: Inverse Reinforcement Learning with Soft Value Iteration Networks for Planetary Rover Path Planning. [23] Hockman B. and Pavone M., (2017) Stochastic motion planning for hopping rovers on small solar system bodies [24] Tamar A., et al (2016) Value iteration networks [25] Ono, M.,et al (2015) Risk-aware planetary rover operation: Autonomous terrain classification and path planning [26] Chiodini, S., et al (2020). Evaluation of 3D CNN Semantic Mapping for Rover Navigation. [27] Phil Stooke (2016), Lunar Farside Landing Plans. [28] Gaudet, B. et al. (2018) Deep Reinforcement Learning for Six Degree-of-Freedom Planetary Powered Descent and Landing. [29] EMC (2012) IDC Digital Universe Study: Big Data, Bigger Digital Shadows and Biggest Growth in the Far East. [30] Vought, R. T. (2019) Memorandum for the heads of executive departments and agencies: Federal Data Strategy - A Framework for Consistency. [31] Open Data NASA, 2020. [32] API Datasource NASA, 2020. [33] Open Access ESA, 2020. [34] Github, [35] Gitlab, [36] Commercial Lunar Payload Services Overview, NASA. [37] J. McCarthy (2006) A proposal for the Dartmouth summer research project on artificial intelligence. [38] M. Montemerlo et al (2006) Winning the DARPA grand challenge with an AI robot. [39] T. Berners-Lee et al (2001) The semantic web. [40] The MIT-IBM Watson AI Lab, IBM. [41] Frontier Development Laboratory (FDL), NASA. [42] Ben Moseley, et al (2020) Unsupervised Learning for Thermophysical Analysis on the Lunar Surface. [43] NVIDIA, Deep Learning for Image Understanding in Planetary Science. [44] Google Brain supported research, Open Sourcing the Hunt for Exoplanets. 